EPJ Web of Conferences 31, 00025 (2012)

DOI: $10.1051 /$ epjconf/20123100025

(C) Owned by the authors, published by EDP Sciences - SIF, 2012

\title{
Evolution of isotopic fission-fragment yields with excitation energy
}

\author{
O. Delaune ${ }^{1}$, M. Caamaño ${ }^{1,2}$, X. Derkx ${ }^{1}$, F. Farget $^{1}$, \\ K.-H. Schmidt ${ }^{3}$, O.B. Tarasov ${ }^{4}$, L. Audouin ${ }^{5}$, A.M. Amthor ${ }^{1}$, \\ C.-O. Bacri ${ }^{5}$, G. Barreau ${ }^{6}$, B. Bastin ${ }^{1}$, D. Bazin ${ }^{4}$, J. Benlliure ${ }^{2}$, \\ B. Blank ${ }^{6}$, L. Cacères ${ }^{1}$, E. Casarejos ${ }^{7}$, \\ B. Fernández-Domínguez ${ }^{2}$, L. Gaudefroy ${ }^{8}$, C. GolabeK ${ }^{1}$, \\ S. Grévy ${ }^{1}$, B. Jurado ${ }^{6}$, O. Kamalou ${ }^{1}$, A. Lemasson ${ }^{1}$, \\ S.M. Lukyanov ${ }^{9}$, W. Mittig ${ }^{4,10}$, D.J. Morrissey ${ }^{4,11}$, A. Navin ${ }^{1}$, \\ J. Pereira ${ }^{4}$, L. Perrot ${ }^{5}$, M. Rejmund ${ }^{1}$, T. Roger ${ }^{1}$, \\ M.G. Saint-Laurent ${ }^{1}$, H. Savajols ${ }^{1}$, C. Schmitt ${ }^{1}$, B.M. Sherrill ${ }^{4}$, \\ C. Stodel ${ }^{1}$, J. TaieB ${ }^{8}$, J.C. Thomas ${ }^{1}$ and A.C.C. Villari ${ }^{12}$
}

${ }^{1}$ GANIL, CEA/DSM-CNRS/IN2P3, BP 55027, F-14076 Caen cedex 5, France

${ }^{2}$ Univ. de Santiago de Compostela, E-15706 Santiago de Compostela, Spain

${ }^{3}$ GSI, Planckstrasse 1, 64291 Darmstadt, Germany

${ }^{4}$ NSCL, Michigan State University, East Lansing, MI 48824, USA

${ }^{5}$ Institut de Physique Nucléaire, CNRS/IN2P3, F-91406 Orsay, France

${ }^{6}$ CENBG, UMR 5797 CNRS/IN2P3, Université Bordeaux 1, F-33175 Gradignan, France

${ }^{7}$ University of Vigo, E-36310, Spain 8 CEA, DAM, DIF, F-91297 Arpajon, France

${ }^{9}$ FLNR, JINR, 141980 Dubna, Moscow region, Russian Federation

${ }^{10}$ Dep. of Physics and Astronomy, Michigan State University, East Lansing, MI 48824, USA

11 Dep. of Chemistry, Michigan State University, East Lansing, MI 48824, USA

12 PANTECHNIK S.A., 13, rue de la Résistance, 14400, Bayeux, France 


\begin{abstract}
Two fission experiments have been performed at GANIL using ${ }^{238} \mathrm{U}$ beams at different energies and light targets. Different fissioning systems were produced with excitation energies from 10 to $230 \mathrm{MeV}$ and their decay by fission was investigated with GANIL spectrometers. Preliminary fission-fragment isotopic distributions have been obtained. The evolution with impinging energy of their properties, the neutron excess and the width of the neutron-number distributions, gives important insights into the dynamics of fusion-fission mechanism.
\end{abstract}

\title{
1 Introduction
}

The binding energy of the nucleus can be estimated in the framework of the liquid-drop model including shell effects and pairing. Assessing the evolution of potential energy with the deformation of a fissioning nucleus, fission-fragment mass yields can be estimated [1]. Up to recently [2], it was impossible to uniquely determine the charge of heavy fission fragments. In the present work, a new experimental technique allowing to measure the complete isotopic distribution in fission reactions is discussed. The production of neutron-rich fission fragments with excitation energy [3] is also investigated.

\section{Experiments}

Two different experiments have been performed at GANIL, using ${ }^{238} \mathrm{U}$ beams at different energies impinging on ${ }^{12} \mathrm{C}$ or ${ }^{9} \mathrm{Be}$ targets. Depending on the impact parameter, different actinides are produced by transfer or fusion reactions, giving access to a broad excitation-energy regime. The fission-fragment distributions of these actinides were investigated using two spectrometers, VAMOS [4] and LISE [5], for the low-energy and high-energy experiments, respectively.

\subsection{Specific features of the experiments}

In the first experiment, the beam impinged on a thin ${ }^{12} \mathrm{C}$ target with an energy of $6.1 \mathrm{~A} \mathrm{MeV}$. At this energy, transfer reactions represent about $10 \%$ of the total cross section [6]. The actinides produced by transfer reactions are tagged by the detection and the identification of the target recoils in a highly segmented annular silicon telescope, SPIDER [7], as shown in figure 1 (a). 

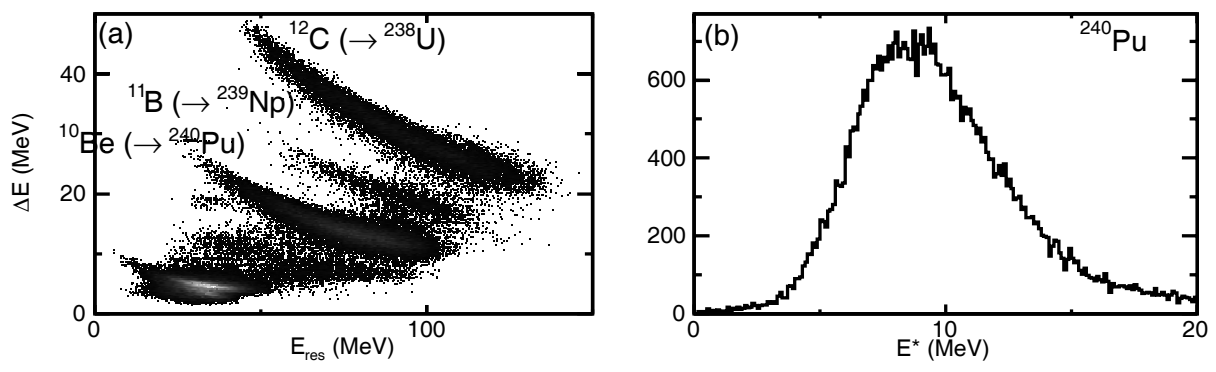

Figure 1: (a) Energy loss $(\Delta \mathrm{E})$ of recoil nuclei as a function of their residual energy $\left(\mathrm{E}_{\mathrm{res}}\right)$. (b) Excitation energy spectrum for ${ }^{240} \mathrm{Pu}$.

For actinides produced by transfer reaction, the excitation energy was computed using two-body kinematics. The excitation energy distribution was measured with a mean value around $9 \mathrm{MeV}$ (see figure 1 (b)). In this work, the two-proton transfer channel, i.e. the production of ${ }^{240} \mathrm{Pu}$, is studied. A tiny beam-energy straggling into the thin target $\left(0.1 \mathrm{mg} / \mathrm{cm}^{2}\right)$ led to the production of compound nuclei in fusion reaction with $E^{*}=45.4 \pm 0.3 \mathrm{MeV}$. In the case that fission occurs, one of the two fission fragments was identified with the VAMOS spectrometer. A second experiment at LISE used a ${ }^{238} \mathrm{U}$ beam of $24 \mathrm{~A} \mathrm{MeV}$. Thick carbon and beryllium targets $\left(15 \mathrm{mg} / \mathrm{cm}^{2}\right.$ for both targets) were used, which resulted in considerable beam-energy straggling. For complete-fusion reactions, the excitation energy of the compound nucleus ranged from 140 to $201 \mathrm{MeV}$ with the beryllium target, and from 209 to $250 \mathrm{MeV}$ for carbon target.

In summary, different fissioning systems with 4 different excitation energies are investigated: ${ }^{240} \mathrm{Pu}$ with $E^{*} \approx 9 \mathrm{MeV}$ (VAMOS), ${ }^{247} \mathrm{Cm}$ with $E^{*} \approx 170 \mathrm{MeV}$ (LISE) and ${ }^{250} \mathrm{Cf}$ with $E^{*} \approx 45 \mathrm{MeV}$ (VAMOS) and $E^{*} \approx 230 \mathrm{MeV}$ (LISE).

\subsection{Identification of fission fragments}

The fission-fragment identification was based in both experiments on the $\mathrm{B} \rho$-ToF-E technique [8], where $\mathrm{B} \rho$ is the magnetic rigidity of the fragment, ToF its time of flight through the spectrometer and E, its total kinetic energy. The atomic number $Z$ identification was obtained from energy-loss measurements, as shown in figure 2 for both experiments.

From the measurement of positions at the focal plane, the path and the radius of curvature, $\rho$, were determined [8]. The measurement of ToF gave the ion velocity, $v$. The radius of curvature is related to the velocity by the 


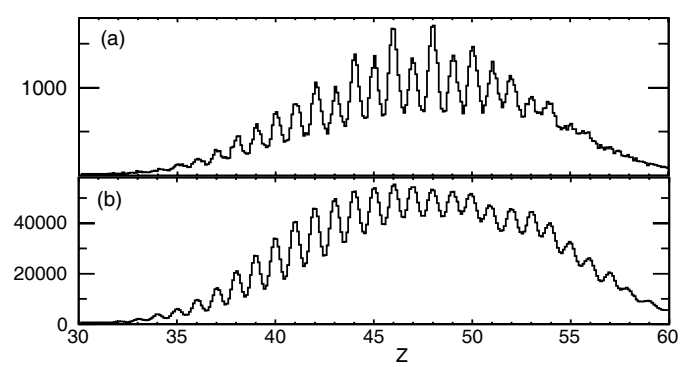

Figure 2: Distribution of the fission-fragment atomic number $Z$ for both experiments (the full data set for ${ }^{250} \mathrm{Cf}$ is presented). The resolutions are $1.6 \%$ for LISE experiment (a) and $1.7 \%$ for VAMOS experiment (b).

relation $B \rho=A v / q$, where $B$ is the magnetic field applied to the dipoles of the spectrometers, $A$, the mass of the ion, and $q$, its ionic charge state. From $B \rho$ and $v$ measurements, the ratio $A / q$ was determined. The mass, $A_{E}$, can also be derived using the velocity and the energy. The ionic charge state is then determined as $q=\frac{A_{E}}{A / q}$ (see figure 3 (a) and (b)). Finally, the mass, $A$, was obtained with $A=A / q \times q$. At VAMOS, a $\gamma$-ray detector located in the target region was used to validate the spectrometer identification [9].
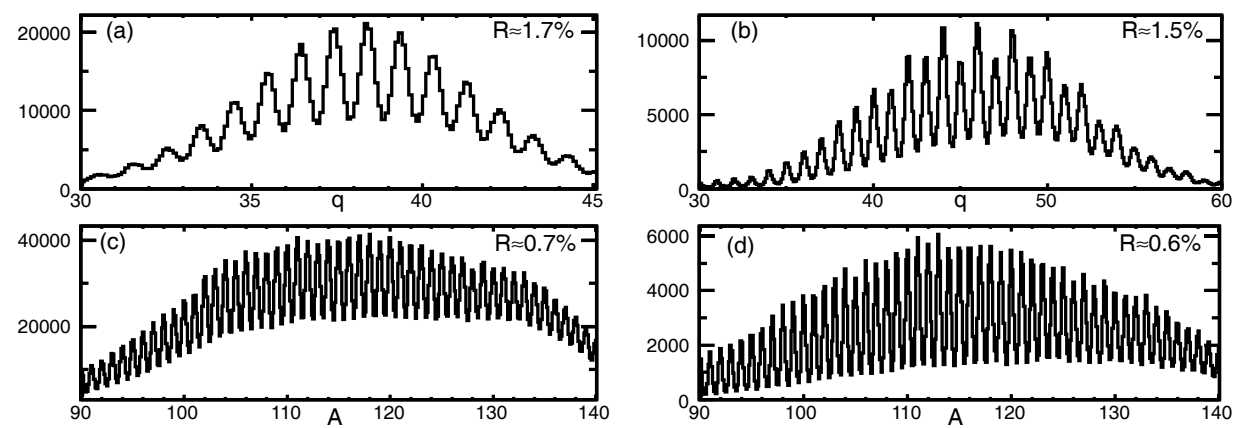

Figure 3: Ionic charge state $q$ and mass $A$ distributions measured from the fission of ${ }^{250} \mathrm{Cf}$ in VAMOS (a) and (c) and in LISE (b) and (d), respectively. Resolutions $R$ are indicated in figures.

\section{$3 \quad$ Fission yields}

To determine isotopic fission yields $Y(Z, A)$, the first step is the reconstruction of the momentum distribution for each ionic charge state, using different $B \rho$ settings of the spectrometer. The different runs are normalised to the 

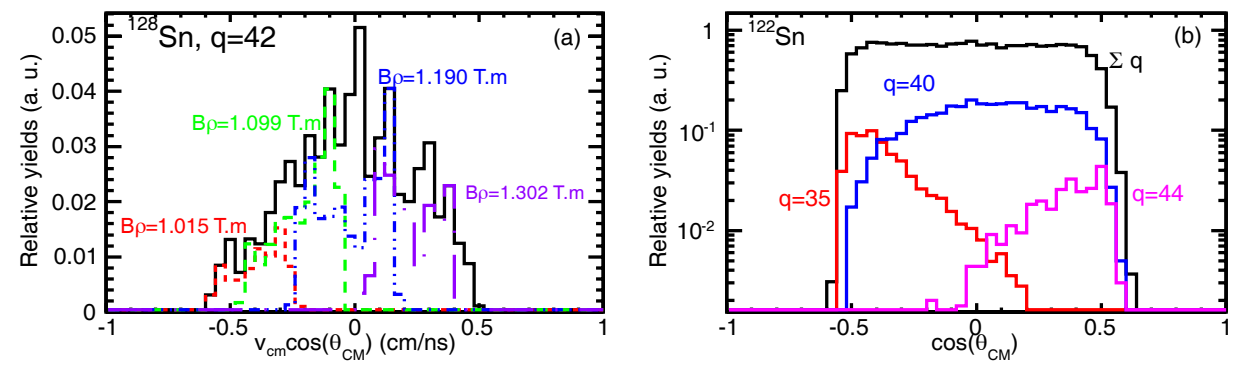

Figure 4: (a) Distribution of the fragment velocity relative to the beam direction for ${ }^{128} \mathrm{Sn}^{42+}$ (colour online). Velocity distributions for different $B \rho$ settings are plotted in colour. The total velocity distribution was obtained taking the envelope of all the settings. (b) Angular distribution in the center of mass $\theta_{C M}$ summing all ionic charge state contributions (colour online).

beam intensity using the elastic scattering of the target in SPIDER in the case of VAMOS experiment. In the case of the LISE experiment, the beam intensity was measured with a Faraday cup before and after each run.

\subsection{Normalisation and spectrometer acceptance correction}

Several $B \rho$ values were needed to cover the full velocity distribution (see figure $4(\mathrm{a}))$. Considering the spectrum envelope, a first estimation of the yield $Y_{0}(Z, A, q)$ for each ion was obtained. The isotopic yield estimation is given by $Y_{1}(Z, A)=\sum_{q} Y_{0}(Z, A, q)$. Finally, $Y_{1}$ was corrected for angular cuts in polar and azimuthal angles, $\theta$ and $\varphi$, using the relation $Y(Z, A)=$ $Y_{1}(Z, A) \frac{2}{\int_{\theta_{\min }}^{\theta_{\max }} \sin \theta d \theta} \int_{\varphi_{\min }}^{\varphi_{\max }} d \varphi \cdot \varphi_{\min }$ and $\varphi_{\max }$ were determined by computing the VAMOS angular acceptance for each event [8]. The angles $\theta_{\min }$ and $\theta_{\max }$ are the observed angular distribution boundaries as shown in figure 4 (b).

\subsection{Preliminary results and discussion}

Following the method described above, isotopic yields were obtained for the four different fissioning systems investigated, and for the complete fragment production. In the following, the main characteristics of the isotopic distributions, namely the neutron excess defined as the ratio of the mean neutron number value $\langle N\rangle$ over $Z$ and the neutron-number width $\sigma(N)$ are studied.

Figure 5 (a) shows the evolution of the mean neutron excess, $\langle N\rangle / Z$, with the fission-fragment atomic number, $Z$, for the different fissioning systems and different excitation energies. At low excitation energy (triangles), light fragments exhibit lower $\langle N\rangle / Z$ values than heavy ones. This can be 

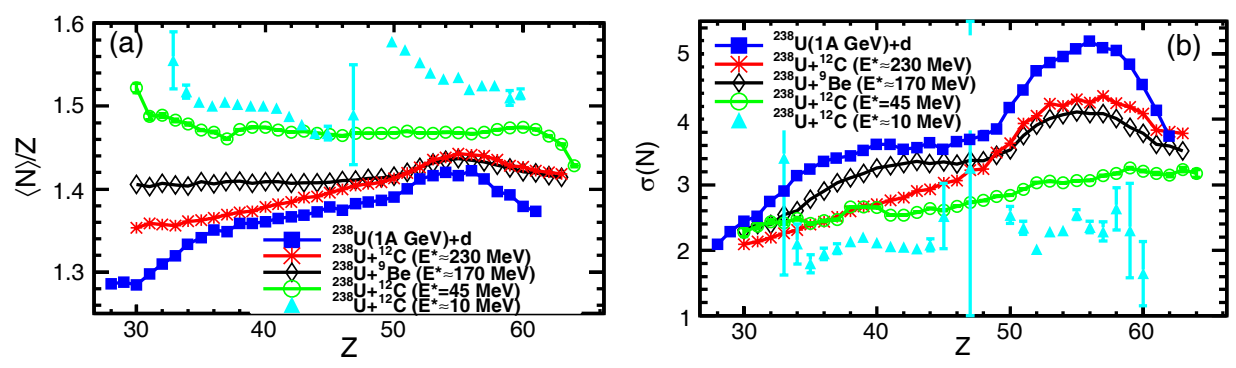

Figure 5: (a) Neutron richness $\langle N\rangle / Z$ as a function of the fission fragments atomic number, $Z$ for different systems (colour online). (b) The width of the neutronnumber distribution $\sigma(N)$ as a function of the fission fragments atomic number, $Z$ for different systems (colour online).

understood as the result of the minimisation of the total potential energy of the emerging fragments: an excess of neutrons in the heavy fragments lowers the influence of the Coulomb energy contribution and symmetry energy in the total potential energy [1]. This trend is enhanced by shell effects around $Z=50$. For the fission of ${ }^{250} \mathrm{Cf}$ produced at $45 \mathrm{MeV}$ (circles), $\langle N\rangle / Z$ gives a constant value of 1.47 , which is consistent with the $N / Z$ ratio of ${ }^{242} \mathrm{Cf}$. This would mean that 8 neutrons in total would be evaporated by the compound nucleus and the fission fragments, independently of the mass of the fission fragment. At higher excitation energy (greater than $170 \mathrm{MeV}$ ) (diamonds and asterisks), the $\langle N\rangle / Z$ ratio decreases as the excitation energy of the compound nucleus increases, indicating more evaporated neutrons. A hump is formed around $Z \approx 54$. This can be understood as the effect of different entrance channels with different excitation energies. At higher bombarding energy, pre-equilibrium particles emission may occur before the formation of the compound nucleus and consequently, the different compound nuclei are produced over a range of $A$ and $E^{*}$. Fission at a low excitation energy induces the hump around $Z \approx 54$ from shell effects which stabilise the mass and atomic-number distributions of heavy fission fragments [10]. This trend is confirmed by data from spallation-fission reactions performed at GSI [11], for ${ }^{238} \mathrm{U}$ at $1 \mathrm{~A} \mathrm{GeV}$ impinging on a deuterium target (squares).

Figure 5 (b) shows the width of the isotopic distribution $\sigma(N)$ as a function of the fission-fragment atomic number for the same fissioning systems. From statistical description of the fission process, $\sigma(N)$ is expected to increase with the temperature $T$ and with the mass $A$ [12], in agreement with the present data. The large energy straggling existing in the highest energy reactions certainly contributes to enlarge the distributions. Likewise 
for $\langle N\rangle / Z$, a large increase of the width is observed around $Z \approx 54$ at high bombarding energy, which most likely reflects the presence of different entrance channels with different excitation energies.

The fragments from the fission of ${ }^{250} \mathrm{Cf}$ produced at $E^{*} \approx 45 \mathrm{MeV}$ show a different behaviour, with constant $\langle N\rangle / Z$ and a regular increase in $\sigma(N)$. In this reaction, shell effects are expected to be weak and entrance-channel effects to be limited. Thus, a liquid-drop behaviour is expected, i.e. $\langle N\rangle / Z$ at scission is expected to increase steadily with $Z$ [13]. The observed constant value of $\langle N\rangle / Z$ with $Z$ suggests that heavy fission fragments evaporate more neutrons than light ones and compensate exactly the neutron excess of fragments at scission. The absence of shell-gap influence, supported by the lack of hump in the evolution of $\sigma(N)$ with $Z$, puts forward that the compound nucleus has no time to evaporate neutrons before reaching the saddle deformation, i.e. first-chance fission is highly probable, as was already suggested in the study of similar fissioning systems at similar excitation energy [14].

\section{Conclusion}

The technique of the inverse kinematics combined with a spectrometer is a powerful tool to investigate fission-fragment distributions. The complete isotopic fission-fragment yields are measured for different fissioning systems at different excitation energies. The production of neutron-rich fission fragments reveals to be a complex process where the influence of the entrance channel effects is decisive. The neutron-excess degree of freedom shows to be very powerful in gaining information about time scales and dynamics of low-energy nuclear reactions. In particular, the present results indicate that at moderate excitation energy the compound nucleus reaches the saddle deformation before any significant cooling by neutron evaporation, and that the fission fragments release the remaining excitation energy.

\section{References}

[1] Wilkins B.D. et al., Phys. Rev. C 14 (1976) 1832.

[2] Schmidt K.-H. et al., Nucl. Phys. A 665 (2000) 221 - 267.

[3] Tarasov O.B. and Villari A.C.C., Nucl. Instr. and Meth. B 266 (2008) 4670 4673.

[4] Savajols H. et al., Nucl. Instr. and Meth. B 204 (2003) 146 - 153. 


\section{EPJ Web of Conferences}

[5] Anne R. et al., Nucl. Instr. and Meth. A 257 (1987) 215 - 232.

[6] Biswas D. C. et al., Phys. Rev. C 56 (1997) 1926 - 1935.

[7] Derkx X. et al., EPJ Web of Conferences, 2010, 2, 07001 CNR09 - Second International Workshop on Compound Nuclear Reactions and Related Topics - Bordeaux, France

[8] Pullanhiotan S. et al., Nucl. Instr. and Meth. A 593 (2008) 343 - 352.

[9] Shrivastava A. et al., Phys. Rev. C 80 (2009) 051305.

[10] Böckstiegel, C. et al., Nucl. Phys. A 802 (2008) 12 - 25.

[11] Pereira J. et al., Phys. Rev. C 75 (2007) 014602.

[12] Yuri T.S. Oganessian and Yuri A. Lazarev, Treatise on Heavy-Ion Science 4, Plenum Press New York, 1985

[13] Berlanger M. et al. Z. Phys. A 291 (1979) 133 - 143

[14] Rubehn Th. et al., Phys. Rev. C 54 (1996) 3062 - 3067. 